\title{
Critical end points in (2+1)-flavor QCD with imaginary chemical potential
}

\author{
Jishnu Goswami* \\ Fakultät für Physik, Universität Bielefeld, D-33615 Bielefeld, Germany; \\ E-mail:jishnu@physik.uni-bielefeld.de
}

\section{Frithjof Karsch}

Fakultät für Physik, Universität Bielefeld, D-33615 Bielefeld, Germany

Physics Department, Brookhaven National Laboratory, Upton, NY 11973, USA

E-mail: karsch@physik.uni-bielefeld.de

\section{Anirban Lahiri}

Fakultät für Physik, Universität Bielefeld, D-33615 Bielefeld, Germany

E-mail: alahiri@physik.uni-bielefeld.de

\section{Marius Neumann}

Fakultät für Physik, Universität Bielefeld, D-33615 Bielefeld, Germany

E-mail: neumann@physik.uni-bielefeld.de

\section{Christian Schmidt}

Fakultät für Physik, Universität Bielefeld, D-33615 Bielefeld, Germany

E-mail: schmidtaphysik.uni-bielefeld.de

\begin{abstract}
We present here the results from an ongoing determination of the critical quark mass in simulations of (2+1)-flavor QCD with an imaginary chemical potential. Studies with unimproved actions found the existence of a critical quark mass value at which the crossover transition ends on a second order phase transition and becomes first order for smaller values of the quark mass for the case of both vanishing and imaginary chemical potential. We use the Highly Improved Staggered Quark (HISQ) action and perform calculations in the Roberge-Weiss (RW) plane, where the value of the critical mass is expected to be largest. The lowest quark mass value used in our simulation corresponds to the pion mass $m_{\pi}$, down to $40 \mathrm{MeV}$. Contrary to calculations performed with unimproved actions we find no evidence for the occurrence of first order transitions at the smallest quark mass values explored so far. Moreover we also show that the chiral observables are sensitive to the RW transition. Our results also indicate that the RW transition and chiral transition could coincide in the chiral limit.
\end{abstract}

Corfu Summer Institute 2018 "School and Workshops on Elementary Particle Physics and Gravity" (CORFU2018)

31 August - 28 September, 2018

Corfu, Greece

\footnotetext{
* Speaker.
} 


\section{Introduction}

The QCD phase diagram at non-zero temperature and baryon chemical potential plays a central role for our understanding and analysis of the properties of strongly interacting matter. Large experimental programs at the Relativistic Heavy Ion Collider (RHIC) in the USA and the Large Hadron Collider (LHC) in Geneva, Switzerland, are devoted to the study of this matter which does undergo a transition from ordinary hadronic matter at low temperatures and densities to a quarkgluon plasma at high temperatures and/or densities.

At physical values of the light and strange quark masses it is now well established that this transition is not a true phase transition but a crossover between two physically distinct regimes. On the other hand, chiral symmetry, which is an exact symmetry in the limit of vanishing values of the quark masses, is known to be spontaneously broken at zero temperature and being restored in the perturbative high temperature limit of QCD. A phase transition thus is expected to occur in the chiral limit, i.e. in the limit of vanishing up and down quark masses, at some critical temperature $T_{c}$.

Using lattice QCD (LQCD) calculations it nowadays is possible to perform non-perturbative calculations with quark masses much smaller than their physical values. A recent LQCD study of $(2+1)$-flavor QCD, i.e. with two light flavors and a strange quark mass fixed to its physical value, performed by the HotQCD Collaboration [1,2], has determined the chiral transition temperature $\left(T_{c} \sim 132 \mathrm{MeV}\right)$ in the chiral limit for the case of vanishing quark chemical potentials $(\mu)$. It also has been shown that the chiral transition is consistent with being a $2^{\text {nd }}$ order transition that is likely to belong to the 3-d $O(4)$ universality class. In more concrete terms no signal for a first order transition has been found down to a pion mass of about $55 \mathrm{MeV}$.

For two massless quark flavors the QCD Lagrangian has an exact $S U(2)_{L} \times S U(2)_{R} \times U(1)_{A} \times$ $U(1)_{V}$ symmetry. However at low temperature, the QCD vacuum is not invariant under the flavor chiral symmetry. As a consequence $S U(2)_{L} \times S U(2)_{R}$ reduces to $S U(2)_{V}$ due to spontaneous symmetry breaking giving rise to three massless Goldstone bosons (pions) in the spectrum. The flavor chiral symmetry gets restored at high temperature $\left(T_{c}\right)$ via a true phase transition in the chiral limit. In-spite of the fact that the $U(1)_{A}$ symmetry is broken by quantum corrections at all temperatures, which causes the mass splitting between the $\eta$ and $\eta^{\prime}$ mesons at low temperature, at very high temperature the quark matter will behave like a free Fermi gas and quantum effects will be negligible; the $U(1)_{A}$ thus will be effectively restored. A crucial question is whether this effective restoration already happens at temperatures close to $T_{C}$ or not. In case, $U(1)_{A}$ is effectively restored already at $T_{c}$, the spontaneous breaking of a larger symmetry group, $U(2)_{L} \times U(2)_{R} / U(2)_{V}$, would be of relevance for the chiral phase transition, which according to the standard scenario put forward by Pisarski and Wilczek [3] may give rise to a first order phase transition, although it has been pointed out that a second order transition also is possible for this larger symmetry group [4, 5].

Studies with unimproved staggered fermions $[6,7,8]$, performed on coarse lattices, suggest that the chiral transition can become $1^{\text {st }}$ order for reasons presumably totally unrelated to the restoration of the anomalous $U_{A}(1)$ symmetry. Below a certain critical quark mass first order transitions have been found, which led to a possible version of the phase diagram in the light and strange quark mass plane (Columbia plot) as shown in Fig. 1 (left). However, in calculations with improved staggered fermion actions, e.g. the Highly Improved Staggered Quark (HISQ) or stout 
actions, which strongly reduce discretization errors, no $1^{\text {st }}$ order transition is found in (2+1) [9] as well as 3-flavor LQCD [10] even for quite small quark masses, corresponding to Goldstone pion masses as small as $50 \mathrm{MeV}$.

If a first order transition exists at smaller quark masses than those currently explored in calculations at $\mu=0$, such a transition would more easily be detected in calculations with a nonvanishing imaginary chemical potential. It has been found in calculations with unimproved staggered fermions that the range of quark masses, for which a $1^{\text {st }}$ order transitions occurs, increases when one performs calculations with a non-zero imaginary chemical potential (see Fig. 1 (right)), i.e. for negative $\mu^{2}$, This $1^{\text {st }}$ order region becomes maximal for $|i \mu / T|=\pi / 3[6,7,8]$. In this work we report on our ongoing studies of the QCD phase diagram with imaginary chemical potential and small values of the light quark masses, keeping the strange quark mass at its physical value. We use the HISQ action as our lattice discretized Dirac operator.
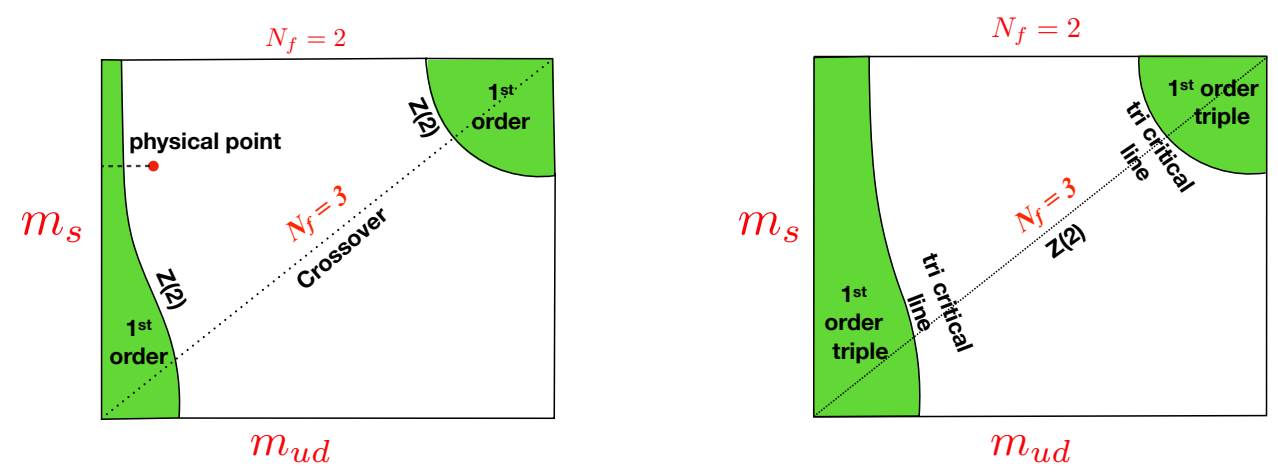

Figure 1: Sketch of possible phases in the light and strange quark mass plane (Columbia plots) for $\mu=0$ (left) and $i \mu / T=i \pi / 3$ (right) planes.

\section{Imaginary chemical potential formalism and Roberge-Weiss (RW) plane}

The QCD partition function with identical imaginary chemical potential, $i \hat{\mu}$, for $f$ quark flavors with mass $m_{f}$ can be written as,

$$
Z(T, V, \mu)=\int \prod_{f} D \psi_{f} D \bar{\psi}_{f} D A_{\mu} \exp \left[-\int_{0}^{\beta} d x_{0} \int_{V} d^{3} x \sum_{f} \bar{\psi}_{f}\left(\gamma_{\mu} D_{\mu}+m_{f}-i \mu \gamma_{0}\right) \psi_{f}-\frac{1}{4} F_{\mu v}^{2}\right] .
$$

In the Euclidean time direction one imposes anti-periodic boundary condition for the quark fields $(\psi(0, \mathbf{x})=-\psi(\beta, \mathbf{x}))$ and periodic boundary condition for the gauge fields $A_{\mu}$. Roberge and Weiss [11] observed that in Eq.(2.1) one can substitute $\psi_{f}\left(x_{0}, \mathbf{x}\right)=\exp \left(i x_{0} \hat{\mu} / \beta\right) \psi_{f}\left(x_{0}, \mathbf{x}\right)$, with $\hat{\mu}=\mu \beta$, which merely results in a change of boundary conditions for the fermion fields,

$$
Z(T, V, \mu)=\int \prod_{f} D \psi_{f} D \bar{\psi}_{f} D A_{\mu} \exp \left[-\int_{0}^{\beta} d x_{0} \int_{V} d^{3} x \sum_{f} \bar{\psi}_{f}\left(\gamma_{\mu} D_{\mu}+m_{f}\right)-\frac{1}{4} F_{\mu v}^{2}\right],
$$

with $\psi\left(x_{0}, \mathbf{x}\right)=\exp (i(\pi+\hat{\mu})) \psi\left(x_{0}+\beta, \mathbf{x}\right)$. They also pointed out that in QCD the partition function is periodic, $Z(\hat{\mu})=Z(\hat{\mu}+2 \pi k / 3)$, which arises from the global $Z(3)$ invariance inherent in Eq.(2.1) and Eq.(2.2). Roberge and Weiss have argued that in a given $Z(3)$ sector, 
$2 \pi k / 3 \leq \hat{\mu} \leq 2 \pi(k+1) / 3$, the partition function is symmetric around the center of this interval, $\mu / T=(2 k+1) \pi / 3$; i.e. an additional $Z(2)$ arises, which may become spontaneously broken at high temperature. The first derivative of free energy $F(T, \hat{\mu}, V)=-T \ln Z(T, \mu, V)$ may become discontinuous at $\mu / T=(2 k+1) \pi / 3$ and a $1^{\text {st }}$ order phase transition may occur for these values of the imaginary chemical potential at high temperature. These particular choices of the imaginary chemical potential in a given $Z(3)$ sector are known as the Roberge-Weiss (RW) planes.

In LQCD formalism one introduces the imaginary chemical potential $(i \mu)$ by multiplying all time-like link fields with a phase, $U_{x, \hat{0}} \rightarrow \exp (i a \mu) U_{x, \hat{0}}, U_{x, \hat{0}}^{\dagger} \rightarrow \exp (-i a \mu) U_{x, \hat{0}}$ [12]. The partition function then still has a real and positive fermion determinant. The gauge fields variables, $U_{x, 0}$, can always be transformed by globally multiplying all time-like gauge field variables with an element of the center group, $z \in\left\{1, \exp \left[ \pm i \frac{2 \pi}{3}\right]\right\}$, i.e. $U_{x, \hat{0}} \rightarrow z U_{x, \hat{0}}$, where $z \in\left\{1, \exp \left[ \pm i \frac{2 \pi}{3}\right]\right\}$. Under this transformation the partition function is again invariant, $Z(T, \hat{\mu}, V)=Z(T, \hat{\mu}+2 \pi / 3, V)$.

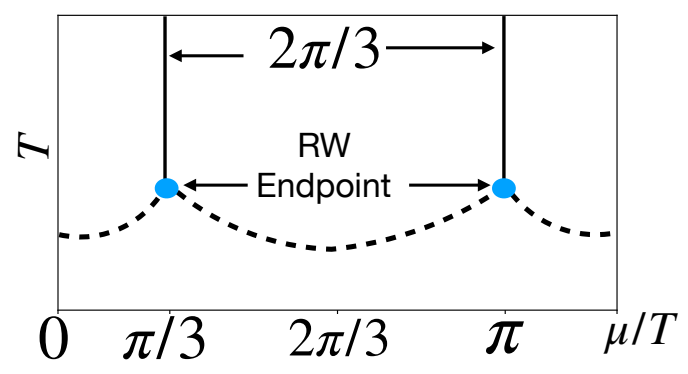

Figure 2: A possible phase diagram in the imaginary chemical potential plane. Solid lines show first order phase transitions, dotted lines correspond to crossover transitions and the blue points indicate $2^{\text {nd }}$ order phase transition points.

As an order parameter for the spontaneous breaking of the $Z(2)$ symmetry in the RW plane the expectation value of the imaginary part of the Polyakov loop is commonly used,

$$
\langle\operatorname{Im} P\rangle=\lim _{\mu \rightarrow \mu_{R W}, V \rightarrow \infty}\left\langle\frac{1}{N_{\sigma}^{3}} \sum_{\mathbf{x}} \operatorname{Im} \operatorname{Tr} \prod_{x_{0}=1}^{N_{\tau}} U_{x, \hat{0}}\right\rangle .
$$

A specific scenario for phase transitions in the RW plane, consistent with the results presented here, is shown in Fig. 2. Alternative scenarios have been found in calculations with standard staggered and also Wilson fermions, where the RW endpoint turns out to be a triple point for sufficiently small values of the light quark masses and three first order transition lines would emerge from this triple point. For $N_{f}=2$ a $1^{\text {st }}$ order triple point has been found on coarse lattices with temporal extent $N_{\tau}=4$ in calculations with standard staggered fermions below $m_{\pi}^{c r i} \sim 400 \mathrm{MeV}$ and with the standard Wilson fermion action below $m_{\pi}^{c r i} \sim 930 \mathrm{MeV}[6,7,8]$. Similar to the case of vanishing chemical potential, results turn out to be quite different in calculations performed with improved actions. No $1^{s t}$ order transition has been found at least for $m_{\pi}>50 \mathrm{MeV}$ in calculations with the stout improved action [9, 13]. Some calculations have also been performed using the 2-flavor HISQ action. In this case no clear-cut results on the order of the phase transition have been reported so far [14]. In our calculations we use the $(2+1)$-flavor HISQ action together with an $\mathscr{O}\left(a^{2}\right)$ improved gauge action to examine the nature of the RW-endpoint at smaller than physical values of the light quark masses and a physical value of the strange quark mass. 


\section{Lattice setup and simulation parameters}

We have performed our calculations with the HISQ action with a temporal extent of $N_{\tau}=4$ and with finite imaginary chemical potential corresponding to the value, $\mu / T=\pi / 3$ for all quark flavors. The constant line of physics is defined according to [15]. It has been obtained by tuning the strange quark mass $\left(m_{s}\right)$ for various values of the gauge couplings in such a way that we obtain the mass of the fictitious pseudo-scalar $\eta_{\bar{s} s}$ meson as $686 \mathrm{MeV}$, which is same as obtained from the Gell-Mann-Okubo mass formula in first order chiral perturbation theory. After tuning the strange quark mass $m_{s}$, the light quark masses $m_{l}$ is chosen such that the $m_{l} / m_{s}$ ratio remains constant for all values of gauge couplings. Different ratios then correspond to different pion masses, e.g. $m_{l} / m_{s}=1 / 27$ corresponds to physical values of the quark masses and gives a pseudo-Goldstone pion mass of about $135 \mathrm{MeV}$. The lowest quark mass ratio used in our calculations, $m_{l} / m_{s}=1 / 320$, corresponds to Goldstone pion mass of about $40 \mathrm{MeV}$. The partition function for $(2+1)$-flavor QCD with two degenerate light quark masses, $m_{l}=\left(m_{u}+m_{d}\right) / 2$, a strange quark mass $\left(m_{s}\right)$ and identical chemical potentials $\mu / T=\pi / 3$ for all flavors may be written as,

$$
Z(T, V, \mu)=\int \mathscr{D} U \operatorname{det}\left[M_{l}(i \mu)\right]^{1 / 2} \operatorname{det}\left[M_{s}(i \mu)\right]^{1 / 4} \exp \left[-S_{G}\right],
$$

where, $M_{f}=D_{H I S Q}(i \mu)+m_{f}$ is the fermion matrix for flavor $f$ and we also use the notation $f \equiv l$ to denote one of the two degenerate light quark flavors. For the gauge action, $S_{G}$, we use the tree level improved Symanzik action.

We use the kaon decay constant $f_{k}$ to set the temperature scale in $\mathrm{MeV}$ [15]. We change the values of the gauge coupling for every ratio $m_{l} / m_{s}$ in such a way that the corresponding temperature varies in the range, $T \sim T_{c} \pm 0.1 T_{c}$. Some further details on our simulation parameters are given in Table 1.

\begin{tabular}{|c|c|c|c|}
\hline$N_{\sigma}$ & $N_{\tau}$ & $m_{l} / m_{s}$ & $m_{\pi}(\mathrm{MeV})$ \\
\hline 8 & 4 & $1 / 27$ & 135 \\
\hline 12 & 4 & $1 / 27$ & 135 \\
\hline 16 & 4 & $1 / 27,1 / 40,1 / 60$ & $135,110,90$ \\
\hline 24 & 4 & $1 / 27,1 / 40,1 / 160,1 / 320$ & $135,110,55,40$ \\
\hline 32 & 4 & $1 / 160$ & 55 \\
\hline
\end{tabular}

Table 1: Details of the numerical simulation parameters for calculations in $(2+1)$-flavor QCD on lattices of size $N_{\sigma}^{3} \times N_{\tau}$.

\section{Results}

\subsection{Nature of the RW endpoint}

All our calculations have been performed in the RW plane, i.e. in the absence of a symmetry breaking field $h=\mu-\mu_{R W}$, that would break explicitly the $Z(2)$ symmetry of the QCD Lagrangian in the RW plane. The appropriate approach for studying the RW phase transition in this case is 
using finite size scaling relations. As the expectation value of the imaginary part of the Polyakov loop vanishes for $h=0$, one usually uses its absolute value as an order parameter,

$$
M \equiv\langle|\operatorname{Im} P|\rangle=\left\langle\left|\frac{1}{N_{\sigma}^{3}} \sum_{\mathbf{x}} \operatorname{Im} \operatorname{Tr} \prod_{x_{0}=1}^{N_{\tau}} U_{x, \hat{0}}\right|\right\rangle .
$$

The finite size scaling relations for the order parameter $M$ and its susceptibility,

$$
\chi_{M}=N_{\sigma}^{3}\left(\left\langle(\operatorname{Im} P)^{2}\right\rangle-\langle|\operatorname{Im} P|\rangle^{2}\right),
$$

are expressed in terms of two universal finite-size scaling functions, $f_{G, L}$ and $f_{\chi, L}$, of the $3-d, Z(2)$ universality class,

$$
\begin{gathered}
M=A N_{\sigma}^{-\beta / v} f_{G, L}\left(z_{f}\right)+r e g . \\
\chi_{M}=B N_{\sigma}^{\gamma / v} f_{\chi, L}\left(z_{f}\right)+r e g .
\end{gathered}
$$

where $z_{f}=z_{0} t N_{\sigma}^{1 / v}$ and $t=\left(T-T_{c}\right) / T_{c}$. These are related to the infinite volume universal scaling functions of the order parameter and its susceptibility, $f_{G}$ and $f_{\chi}$, respectively [16]. We also calculate the Binder cumulant $[17,18] B_{4}$ which is given in terms of the ratio of fourth and second order moments of the order parameter,

$$
B_{4}(T, V)=\frac{\left\langle(\operatorname{Im} P)^{4}\right\rangle}{\left\langle(\operatorname{Im} P)^{2}\right\rangle^{2}}
$$

Close to the critical point this ratio also is expressed in terms of a universal ratio of scaling functions, $B_{4}(T, V)=f_{B}\left(z_{f}\right)+$ reg..

For our finite-size scaling analysis we have calculated the finite-size scaling functions using the $\lambda \phi^{4}$ spin model with a tuned parameter $\lambda$ such as to reduce contributions arising from correctionsto-scaling (improved Ising model). This $Z(2)$ invariant spin model has been used previously to determine the infinite volume scaling functions of the 3- $d$ Ising model [16].

In order to test universality of several observables obtained from our $(2+1)$-flavor QCD calculations with an imaginary chemical potential we only fit the order parameter with the universal scaling function $f_{G, L}$. This fit yields results for the non-universal parameters $T_{c}, A$ and $z_{0}$. We use these parameters to compare other observables, e.g. $\chi_{M}$ and $B_{4}$ to the corresponding scaling functions $f_{\chi}, f_{B}$. These comparisons are parameter free, when taking into account that the amplitude $B$, appearing in Eq. 4.4, can be expressed in terms of the amplitude $A$ in Eq. 4.3, i.e. $B=A^{2}$.

In Fig. 3 we show the order parameter $M$, its susceptibility $\chi_{M}$ and the Binder cumulant $B_{4}$, calculated on lattices with different spatial extent, $N_{\sigma}$, and for the physical light to strange quark mass ratio $m_{l} / m_{s}=1 / 27$. Also shown are fits to $M$ based on the ansatz given in Eq. 4.3 and the resulting finite-size scaling curves for $\chi_{M}$. The order parameter data follow the universal scaling curve nicely in the explored temperature range. For the susceptibility, which is not fitted, scaling violations are clearly visible in the symmetry broken phase for the smaller volumes, while scaling violations seem to be small in the symmetric phase. A similar behavior also is found for the Binder cumulant $B_{4}$. The significance of scaling violations on small lattices also is apparent from the rescaled observables shown in Fig. 4. 

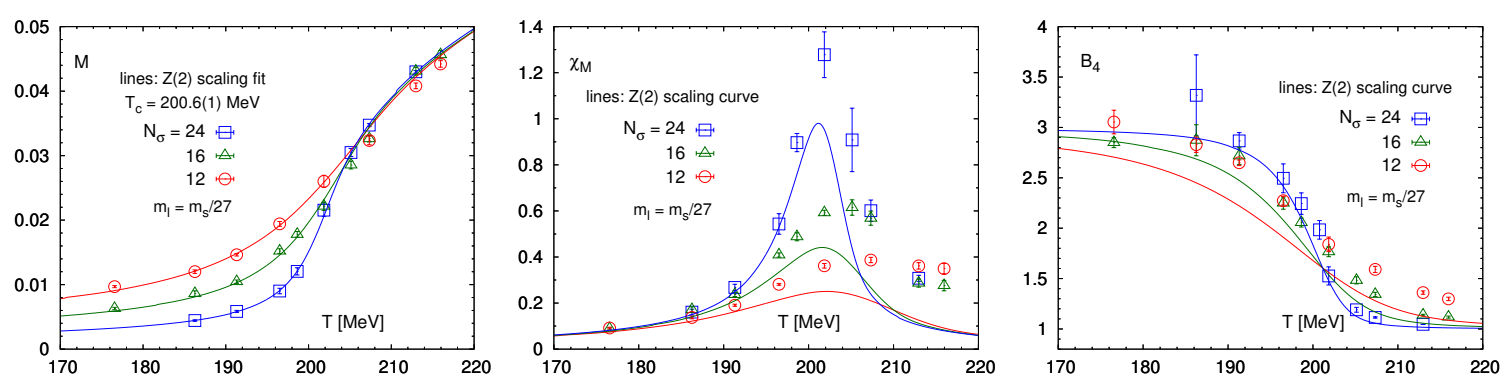

Figure 3: The order parameter $M$ (left), its susceptibility $\chi_{M}$ (middle) and the Binder cumulant $B_{4}$ (right) for $m_{l} / m_{s}=1 / 27$ and various lattice sizes. Lines are based on a fit of $M$ to the $Z(2)$ finite-size scaling function $f_{G, L}$. Curves shown for $\chi_{M}$ and $B_{4}$ are then parameter free results of this fit.
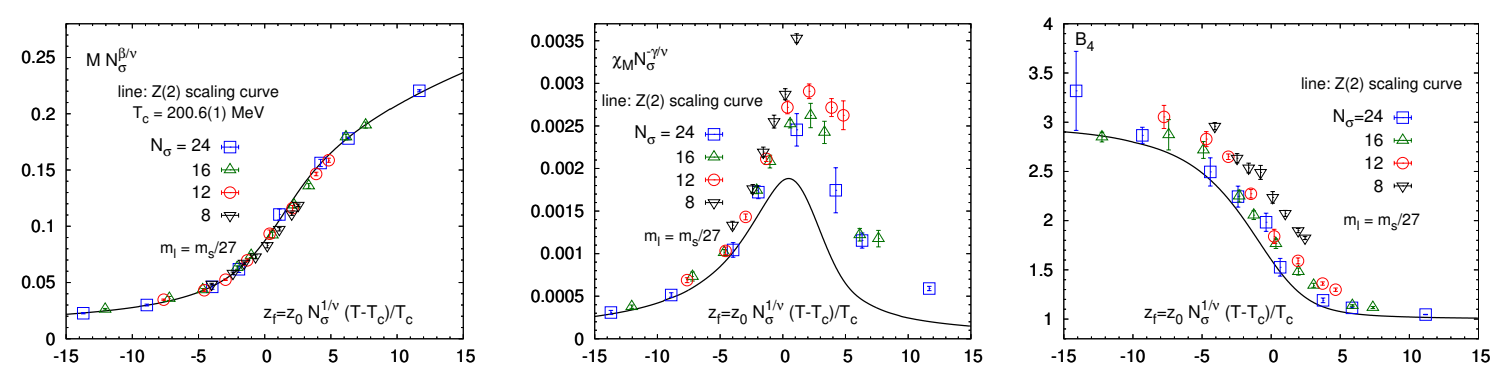

Figure 4: The rescaled order parameter $M$ (left), the rescaled susceptibility $\chi_{M}$ (middle) and the rescaled Binder cumulant $B_{4}$ (right) for $m_{l} / m_{s}=1 / 27$ and various lattice sizes. Lines show the corresponding 3-d, $Z(2)$ finite size scaling functions.

When changing the spatial extent, $N_{\sigma}$, of the lattice, the peak height of the order parameter susceptibility would increase proportional to volume, $V \equiv N_{\sigma}^{3}$, if the phase transition at the RW endpoint would be first order in the infinite volume limit. For a second order phase transition in the $3-d, Z(2)$ universality class the peak height only increases as $N_{\sigma}^{\gamma / v}=N_{\sigma}^{1.96}$. As can be seen from the rescaled susceptibility, Fig. 4 (middle), our results for $m_{l} / m_{s}=1 / 27$ are consistent with this slower rise. We thus find that the RW endpoint for this quark mass ratio still corresponds to a $2^{\text {nd }}$ order phase transition in the Ising universality class.

Similar behavior we find in all our calculations with light quark masses down to $m_{l}=m_{s} / 320$. The non-universal scaling parameters, of course, will be quark mass dependent. Indeed, we find that the phase transition temperature decreases slightly with quark mass, although we cannot yet

\begin{tabular}{|c|c|c|c|}
\hline$m_{l} / m_{s}$ & $m_{\pi}$ & $T_{c}$ & $\Delta T_{c}$ \\
\hline $1 / 27$ & 135 & 200.6 & 0.18 \\
\hline $1 / 40$ & 110 & 199.37 & 0.23 \\
\hline $1 / 160$ & 55 & 195.23 & 0.27 \\
\hline $1 / 320$ & 40 & 195.36 & 0.29 \\
\hline
\end{tabular}

Table 2: Phase transition temperature, $T_{c}$, at the endpoint of the line of $1^{s t}$ order transitions in the RW plan. Results are obtained on lattices with temporal extent $N_{\tau}=4$ an different quark mass ratios $m_{l} / m_{s}$. 

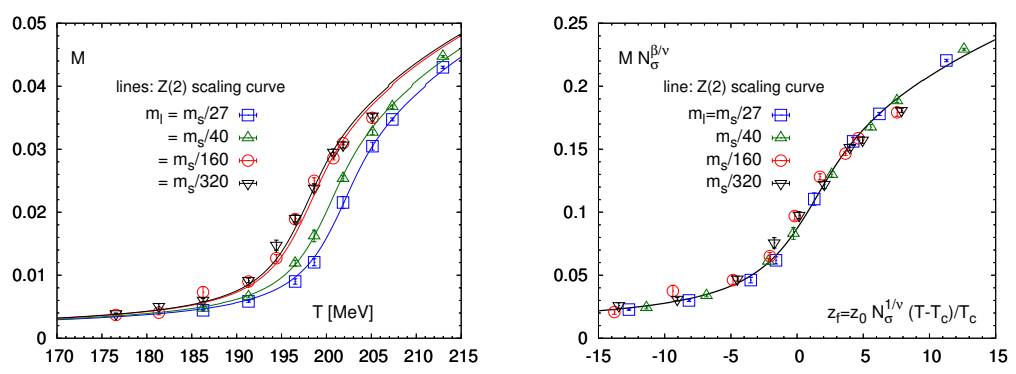

Figure 5: The order parameter $M$ for various $m_{l} / m_{s}$ ratios for a fixed volume $N_{\sigma}=24$. Lines correspond to fits using the $\mathrm{Z}(2)$ finite size scaling functions. The corresponding rescaled data are shown in the figure on the right.

specify in detail the functional form of the dependence on $m_{l} / m_{s}$. Results for the phase transition temperature at different values of $m_{l} / m_{s}$ are summarized in Table 2.

Results obtained for the order parameter $M$ on lattices with spatial extent $N_{\sigma}=24$ and for four different values of the light quark masses are shown in Fig. 5 (left). The shift of the crossover region to smaller values of the temperature is obvious, while the shape of the overall temperature dependence does not show any significant modification, indicating that any quark mass dependence of the non-universal amplitude $A$ and the scale factor $z_{0}$ is small. In fact, we do not find any systematics for the quark mass dependence of these parameters. As can be seen in Fig. 5 (right) the rescaled order parameter in all cases agrees well with the universal 3- $d, Z(2)$ scaling function $f_{G, L}\left(z_{f}\right)$, giving further support to the assertion that the RW phase transition is $2^{\text {nd }}$ order in the entire interval of quark masses considered by us.

\subsection{Chiral phase transition in RW plane}

Let us now turn to a discussion of the chiral phase transition in $(2+1)$-flavor QCD at nonzero imaginary chemical potential. In the chiral limit the chiral phase transition occurs alongside with the RW transition. The order parameter for this transition, the renormalization group invariant (RGI) chiral condensate, can be defined as,

$$
\Delta_{l s}=\frac{2}{f_{K}^{4}}\left(m_{s}\langle\bar{\psi} \psi\rangle_{l}-m_{l}\langle\bar{\psi} \psi\rangle_{s}\right)
$$

where

$$
\langle\bar{\psi} \psi\rangle_{f}=\frac{T}{V} \frac{\partial \ln Z\left(T, V, m_{f}, \mu\right)}{\partial m_{f}}=\frac{1}{N_{\sigma}^{3} N_{\tau}}\left\langle\operatorname{Tr}_{\mathrm{f}}^{-1}\right\rangle .
$$

We used the kaon decay constant $f_{K}$ for normalization of the dimensionless order parameter $\Delta_{l s}$. For the determination of a pseudo-critical temperature, $T_{\chi}$, for chiral symmetry restoration we use the fluctuations of the light quark chiral condensate,

$$
\chi^{\text {disc }}=4 \frac{m_{s}^{2}}{N_{\sigma}^{3} N_{\tau}}\left(\left\langle\left(\operatorname{Tr}_{1}^{-1}\right)^{2}\right\rangle-\left\langle\left(\operatorname{Tr}_{1}^{-1}\right)\right\rangle^{2}\right) / f_{k}^{4} .
$$

In Fig. 6 we show the order parameter $\Delta_{l s}$, its temperature derivative $T d \Delta_{l s} / d T$, and the disconnected chiral susceptibility $\chi^{\text {disc }}$ for the light quark mass, $m_{l}=m_{s} / 160$. The weak volume dependence found for the peak height of $T d \Delta_{l s} / d T$ as well as $\chi^{\text {disc }}$ rules out the possibility of a first 
order RW transition in the chiral observables. This is consistent with the conclusion from the previous section. Nonetheless, the peak heights continue to increase with increasing volume also on the largest lattices available to us. This suggests that the chiral susceptibilities at non-zero values of the quark mass, are sensitive to the RW transition. In fact, they are expected to behave like temperature like observables in an effective $Z(2)$ symmetric Hamiltonian for the RW transition. The slow rise of the peak heights is consistent with this assertion. Also the location of the peak of $\chi^{\text {disc }}, T_{\chi}$ is consistent with the phase transition temperature, $T_{c}$, of the RW transition. This suggests that also in the chiral limit the RW and chiral transition may coincide and occur at the same temperature. However, a more detailed analysis of such a scenario is clearly needed.
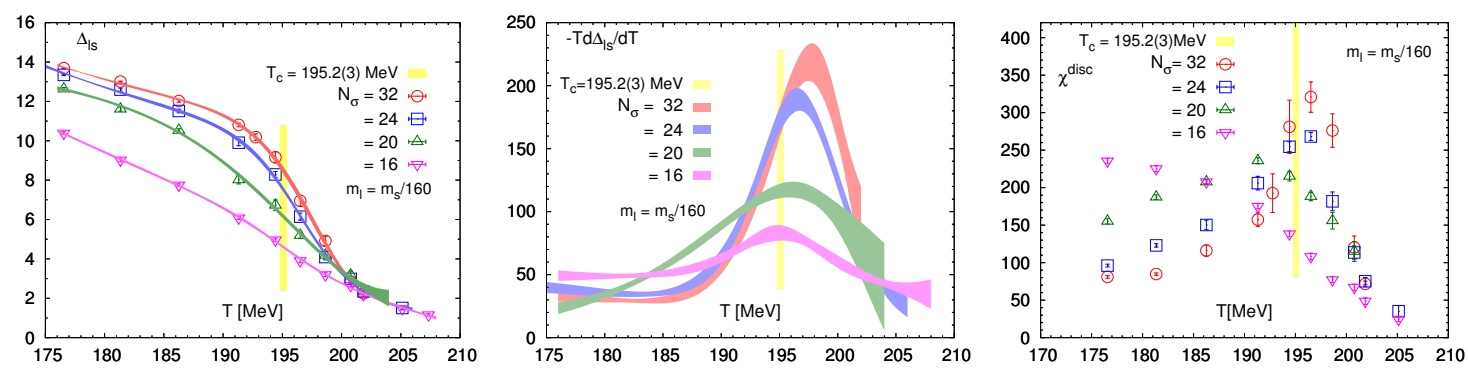

Figure 6: The RGI chiral condensate, $\Delta_{l s}$ (left), its temperature derivative (middle), and the disconnected chiral susceptibility (right) versus temperature for various spatial lattice sizes and fixed quark mass ratio $m_{l} / m_{s}=1 / 160$. The yellow bands show the location of the RW transition temperature extracted from the location of the peak of the order parameter susceptibility $\chi_{M}$.

\section{Conclusions}

We presented here some results from our ongoing studies of the phase transition in $(2+1)$ flavor QCD with imaginary chemical potential in the RW plane. Our finite size scaling analysis with the HISQ action, performed on lattices with temporal extent $N_{\tau}=4$, show that the phase transition in the RW plane is $2^{\text {nd }}$ order belonging to the 3- $d, Z(2)$ universality class for physical values of the quark masses down to small quark masses, corresponding to a Goldstone pion mass of about $40 \mathrm{MeV}$. At present we see no indication for a possible $1^{\text {st }}$ order transition that may occur in the chiral limit. However, the nature of the chiral phase transition itself still requires further analysis. Current results indicate that it may coincide with the RW transition also in the chiral limit.

\section{Acknowledgement}

This work was supported in part through Contract No. DE-SC001270 with the U.S. Department of Energy, the Deutsche Forschungsgemeinschaft (DFG) through the CRC-TR 211 "Stronginteraction matter under extreme conditions", grant number 315477589 - TRR 211, and the grant 05P18PBCA1 of the German Bundesministerium für Bildung und Forschung.

\section{References}

[1] H. T. Ding, P. Hegde, F. Karsch, A. Lahiri, S. T. Li, S. Mukherjee et al., Chiral phase transition of (2+1)-flavor QCD, Nucl. Phys. A982 (2019) 211 [1807.05727]. 
[2] H. T. Ding et al., The chiral phase transition temperature in (2+1)-flavor QCD, 1903.04801.

[3] R. D. Pisarski and F. Wilczek, Remarks on the Chiral Phase Transition in Chromodynamics, Phys. Rev. D29 (1984) 338.

[4] A. Pelissetto and E. Vicari, Relevance of the axial anomaly at the finite-temperature chiral transition in QCD, Phys. Rev. D88 (2013) 105018 [1309. 5446 ].

[5] J. Eser, M. Grahl and D. H. Rischke, Functional Renormalization Group Study of the Chiral Phase Transition Including Vector and Axial-vector Mesons, Phys. Rev. D92 (2015) 096008 [1508.06928].

[6] P. de Forcrand and O. Philipsen, Constraining the QCD phase diagram by tricritical lines at imaginary chemical potential, Phys. Rev. Lett. 105 (2010) 152001 [10 04 . 3144 ].

[7] O. Philipsen and C. Pinke, The $N_{f}=2 Q C D$ chiral phase transition with Wilson fermions at zero and imaginary chemical potential, Phys. Rev. D93 (2016) 114507 [1602.06129].

[8] C. Czaban, F. Cuteri, O. Philipsen, C. Pinke and A. Sciarra, Roberge-Weiss transition in $N_{f}=2 Q C D$ with Wilson fermions and $N_{\tau}=6$, Phys. Rev. D93 (2016) 054507 [1512. 07180].

[9] C. Bonati, E. Calore, M. D’Elia, M. Mesiti, F. Negro, F. Sanfilippo et al., Roberge-Weiss endpoint and chiral symmetry restoration in $N_{f}=2+1 Q C D, 1807.02106$.

[10] A. Bazavov, H. T. Ding, P. Hegde, F. Karsch, E. Laermann, S. Mukherjee et al., Chiral phase structure of three flavor QCD at vanishing baryon number density, Phys. Rev. D95 (2017) 074505 [1701.03548].

[11] A. Roberge and N. Weiss, Gauge Theories With Imaginary Chemical Potential and the Phases of QCD, Nucl. Phys. B275 (1986) 734.

[12] P. Hasenfratz and F. Karsch, Chemical Potential on the Lattice, Phys. Lett. 125B (1983) 308.

[13] C. Bonati, M. D’Elia, M. Mariti, M. Mesiti, F. Negro and F. Sanfilippo, Roberge-Weiss endpoint at the physical point of $N_{f}=2+1$ QCD, Phys. Rev. D93 (2016) 074504 [1602.01426].

[14] L.-K. Wu and F.-L. Zhang, Nature of the chiral phase transition of two flavor QCD from an imaginary chemical potential with HISQ fermions, Phys. Rev. D97 (2018) 114514 [1802. 00773 ].

[15] A. Bazavov et al., The chiral and deconfinement aspects of the QCD transition, Phys. Rev. D85 (2012) 054503 [1111.1710].

[16] J. Engels, L. Fromme and M. Seniuch, Numerical equation of state from an improved three-dimensional Ising model, Nucl. Phys. B655 (2003) 277 [cond-mat/ 0209492 ].

[17] K. Binder, Finite size scaling analysis of Ising model block distribution functions, Z. Phys. B43 (1981) 119.

[18] K. Binder, Critical Properties from Monte Carlo Coarse Graining and Renormalization, Phys. Rev. Lett. 47 (1981) 693. 